\title{
Influence of chronotype on daily mood fluctuations: pilot study in patients with depression
}

\author{
Konstantin F. Brückmann, Jürgen Hennig, Matthias J. Müller, Stanislava Fockenberg, Anne-Marthe Schmidt, \\ Nicole Cabanel and Bernd Kundermann
}

\section{Summary}

Depression risk is associated with a late chronotype pattern often described as an 'evening chronotype'. Fluctuations in mood over consecutive days have not yet been measured according to chronotype in in-patients with depression. A total of 30 in-patients with depression and 32 healthy controls matched for gender and age completed a chronotype questionnaire and twice-daily ratings on mood for 10 consecutive days (registered in the German Clinical Trials Register: DRKS00010215). The inpatients had Saturdays and Sundays as hospital-leave days. The relationship between chronotype and daily mood was mediated by the weekday-weekend schedule with higher levels of negative affect in the evening-chronotype patient subgroup at weekends. Results are discussed with respect to a probably advantageous standardised clinical setting with early morning routines, especially for patients with evening chronotypes.

\section{Declaration of interest}

None.

\section{Keywords}

Chronotype; depression; daily mood; week-weekenddifferences.

\section{Copyright and usage}

(c) The Royal College of Psychiatrists 2020. This is an Open Access article, distributed under the terms of the Creative Commons Attribution-NonCommercial-NoDerivatives licence (http://creative commons.org/licenses/by-nc-nd/4.0/), which permits noncommercial re-use, distribution, and reproduction in any medium, provided the original work is unaltered and is properly cited. The written permission of Cambridge University Press must be obtained for commercial re-use or in order to create a derivative work.
With respect to circadian functionality, individuals differ as to optimal time of day. Those with a preference for activities in the morning are known as 'morning chronotypes' whereas those with preferences for evening activities are characterised as 'evening chronotypes' (see for example Mueller \& $\mathrm{Haag}^{1}$ ). The majority of individuals are intermediate in this respect. As early as 1991, Drennan and co-workers showed that patients with depression are mainly characterised with evening chronotypes; ${ }^{2}$ however, the studies relating chronotype to a clinical diagnosis of depression are inconsistent. ${ }^{3}$ More specifically, 'eveningness' seems to relate to the severity of the disease as well as indicated by a higher occurrence of suicidal thoughts. ${ }^{4}$ A recent prospective study demonstrated that evening chronotype measured in adolescence predicted the occurrence of depressive episodes 1 year later. ${ }^{5}$ However, beside stability and moderate heritability of the individual chronotype, variance in overt behaviour can be observed due to different factors. There is a clear shift with age, chronotype moving later in adolescence and switching to earlier after 20, gender differences and seasonal modulation. ${ }^{6}$ Within a given chronotype time periods of activity can markedly differ according to work or leisure time. ${ }^{7}$ Even a treatment such as cognitive-behavioural therapy, can move patients with depression toward more 'morningness'. Thus, day-to-day changes in activity preferences as a result of social cues and the seasonal, and the therapeutic modifiability of the otherwise relatively stable chronotype point to a dynamic and complex synchronisation process.

As circadian mood and activity fluctuations are among the core symptoms of depression (at least in 'melancholic' subtypes) we investigated whether patients with depression differ according to their chronotype in consecutive twice-daily mood assessments on weekdays and weekends. For this kind of research question a standardised setting is optimal because the time points of measurements (such as mood ratings) can be planned and controlled. We, therefore, studied only in-patients with depression. We expected interactions between chronotype and weekends (corresponding to leisure time in healthy samples) with the pattern of more negative affect in the morning occurred in those with evening chronotypes and more negative affect in the evening in those with morning chronotypes. For weekdays this pattern will be masked because of therapeutic sessions or other reasons.

\section{Method}

A total number of $n=30$ patients (all diagnosed with ICD-F32, ${ }^{9}$ in particular F32.2) between 18 and 50 years old (mean 32.2, s.d. $=8.4$, 17 men and 13 women) were compared with a healthy control group of 13 men and 19 women (mean age 32.0, s.d. $=8.3$ ). All participants were fully informed about the study and gave informed written consent. All patients had hospital leave on Saturday and Sunday. This study was part of a larger research project (sleep, circadian rhythmicity, mood and pain perception in patients with affective and affect regulation disorders and healthy controls) and was approved by the ethics committee of the medical faculty of the Justus-Liebig-University of Giessen, Germany. The study is registered in the German Clinical Trials Register (DRKS00010215).

Patients were not entirely free from medication. Treatment consisted of selective serotonin reuptake inhibitors $(n=14)$, Serotoninnoradrenaline reuptake inhibitors $(n=4)$, agomelatine $(n=3)$, tricyclic antidepressants $(n=1)$ or no medication $(n=8)$. Patients had a mean Beck Depression Inventory (BDI-II) ${ }^{10}$ score of 26.5 (s.d. $=10.8)$.

Participants completed the German version of the 'MorningnessEveningness Questionnaire' (MEQ), which has been demonstrated to be reliable and valid. ${ }^{11}$ The proportion of patients and controls in chronotype categories based on Horne \& Östberg ${ }^{12}$ with only three categories (moderate evening chronotypes + definite evening chronotype; intermediate; moderate + definite morning chronotype) of the MEQ were: evening type (range 16-41): 5 in the patient group and 5 in the control group; intermediate type 
(range 42-58): 19 in the patient group and 16 in the control group; and morning type (range 59-86): 6 in the patient group and 11 in the control group. As frequencies especially for the evening chronotype were fairly low, we classified individuals into morning chronotype or evening chronotype according to median split of the MEQ within each group but we also show our results for the three-group classification.

Subjective well-being was measured by a Likert-scale with 14 items having loadings on three factors: negative affect, lack of energy and well-being. ${ }^{13}$ The present study primarily relates to 'negative affect' although 'lack of energy' will be used to demonstrate the validity of the grouping used for chronotype. Ratings on emotional states were obtained in the morning $(07.00-09.00 \mathrm{~h})$ and in the evening (18.00-20.00 h) starting on Tuesday evening until Thursday 1 week later. Results were calculated only for the 2 days before the weekend, 2 weekend-days and 2 days after the weekend (in the morning and evening, respectively) in order to guarantee that all participants had become familiar with the procedure, which could increase reliability. The design, therefore, consists of two independent factors with two levels each (patients/controls and morning chronotype/evening chronotype) and two within factors with three levels for schedule (weekdays prior weekend/ weekend/weekdays after weekend) and two levels for time of day (morning/evening). Data were, therefore, analysed with analysis of variance for repeated measures.

\section{Results}

We were able to demonstrate the validity of the MEQ and the way we defined evening chronotype and morning chronotype. A main effect across all time points of measurement and both groups (patients and controls) revealed a higher lack of energy in those with morning chronotypes in the evening and in those with evening chronotypes in the morning $(F=5.7$, d.f. $=1,58, P$ $<0.05$ ). Within-patients chronotype did not relate to severity of disease in our participants as indicated by non-significant differences in BDI (data not shown, $t(30)=-0.2, P=0.8$ ). Moreover, different treatment or gender was not related to chronotype (data not shown).

Regarding 'negative affect' a significant three-way-interaction $(F=4.5$, d.f. $=2,57, P<0.05)$ between group, chronotype and schedule occurred indicating different mood states depending on chronotype and week-weekend schedule especially in patients. These results are nearly identical when excluding the three patients with melatonergic medication $(F=4.63$, d.f. $=2,53, P<$ $0.05)$. Additionally, we looked at the results based on the categorial classification. ${ }^{12}$ These are in the same direction but failed to reach significance. For a direct comparison of the two approaches see Fig. 1.

Figure 1a indicates that patients with a late chronotype (evening chronotypes) got worse at weekends whereas those with a early chronotype improved. No systematic differences could be statistically confirmed in the healthy control group. In contrast, in patients post hoc tests (for dependent samples) reveal that especially those with morning chronotypes significantly improved at weekends $(t(31)=2.1, P<0.05)$ whereas evening chronotypes may get worse at weekends but this contrast did not reach significance. Based on a categorial grouping into evening chronotypes, intermediate and morning chronotypes, the direction of effects is similar but fails to reach statistical significance (presumably because of low numbers in the evening- chronotype and morning-chronotype subgroups).
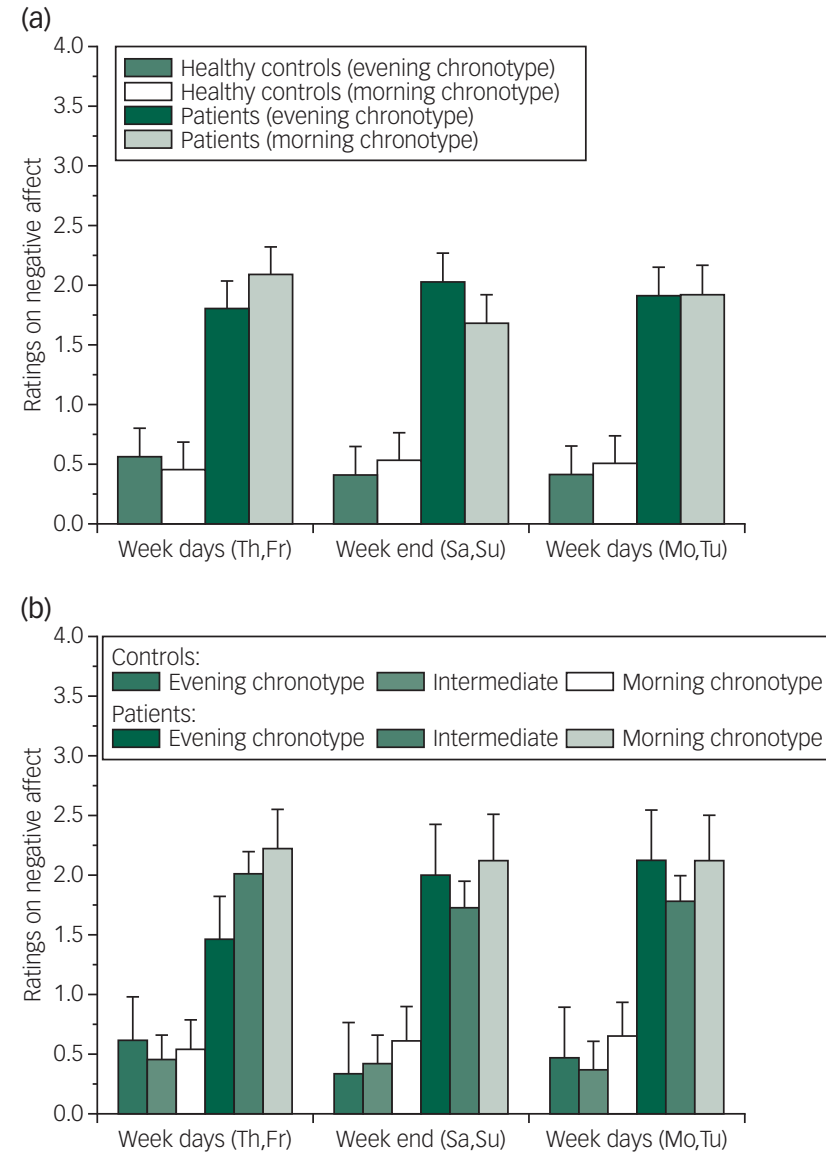

Fig. 1 Negative affect rating in the in-patient and control groups by chronotype and schedule.

Means and standard errors (s.e.m.) of self ratings on 'negative affect' depending on Morningness-Eveningness Questionnaire (MEQ) scores divided by (a) median, split into evening type (ET) and morning type (MT), and (b) categorical classification, according to Horne and Ostberg ${ }^{10}$, split into ET, MT and intermediate type in depressed patients and healthy controls. Note that dichotomisation by median split reached statistical significance while categorisation into three groups did not due to lower sample sizes in each group (see text).

Th, Thursday; Fr, Friday; Sa, Saturday; Su, Sunday; Mo, Monday; Tu, Tuesday.

\section{Discussion}

Our results demonstrate that the concept of chronotype may go beyond different preferences and/or activities in the morning or evening. In general, we were able to show that individuals with evening chronotypes complain about less energy in the morning, whereas those with morning chronotypes feel less energetic in the evening. The notion that severity of depression may be more pronounced in patients with a late chronotype could not be confirmed in our study. A direct comparison of BDI-II scale values in patients with depression between those with evening chronotypes and morning chronotypes only indicates somewhat higher values in the evening-chronotype subgroup but this difference did not reach statistical significance.

Our main finding is that the morning chronotype subgroup improved during weekends whereas ratings on negative affect rose in those in-patients in the evening-chronotype subgroup. Note, that all patients had hospital leave on Saturdays and Sundays. Ratings on sleep quality obtained daily for the whole study period did not differ by chronotype (data not shown, so different sleep quality cannot account for the results obtained on mood). 
A recent study in patients with bipolar disorders demonstrates that a combination of sleep deprivation and light therapy results in significant decreases in depression symptoms with a better improvement in those with evening chronotypes compared with morning chronotypes or intermediate types. ${ }^{14}$ It can be speculated that individuals with evening chronotypes in our study may have an advantage in a clinical setting with therapeutic regimens starting in the early morning. The need to get up early may, therefore, result in (partial) sleep deprivation in those with evening chronotypes, which does not take place at weekends. However, further studies with a larger sample size and grouping into evening chronotypes and morning chronotypes based on the categorial approach ${ }^{12}$ have to address this question explicitly.

The fact that suicide attempts are more frequent and mediated by depression in individuals with evening chronotypes ${ }^{15}$ means the inclusion of chronotype in the diagnostic process can be recommended. ${ }^{16}$ If the finding that in-patients with evening chronotypes probably worsen during weekend hospital leave can be replicated in larger samples (sample size is of course a limitation of our pilot study), special attention is required as many studies demonstrated that suicides peak on Mondays. ${ }^{17}$

Konstantin F. Brückmann (D. BSC, Department of Psychology and Sports Science Center for Psychobiology and Behavioral Medicine, Justus-Liebig-University; and Clinic for Psychiatry and Psychotherapy, VITOS, Germany; Jürgen Hennig, PhD, Department for Psychiatry and Psychotherapy, VITOS, Germany; Jürgen Hennig, PhD, Department
Psychology and Sports Science, Center for Psychobiology and Behavioral Medicine, Justus-Liebig-University, Germany; Matthias J. Müller, MD, PhD, Oberberg Group; and Faculty of Medicine, Justus-Liebig University, Germany; Stanislava Fockenberg, MD, Clinic for Psychiatry and Psychotherapy, VITOS, Germany; Anne-Marthe Schmidt, MD, Clinic for Psychiatry and Psychotherapy, VITOS, Germany: Nicole Cabanel, MD, Clinic for Psychiatry and Psychotherapy, VITOS; and Department of Psychiatry and Psychotherapy,

Philipps-University Marburg; Bernd Kundermann, PhD, Clinic for Psychiatry and

Philipps-University Marburg; Bernd Kundermann, PhD, Clinic for Psychiatry and
Psychotherapy, VITOS; and Department of Psychiatry and Psychotherapy, PhilippsUniversity Marburg, Germany

Correspondence: Konstantin Falk Brückmann. Email: k.brueckmann@hotmail.de

First received 2 May 2019, final revision 21 Nov 2019, accepted 21 Dec 2019

\section{Acknowledgements}

We thank one anonymous reviewer for the constructive and helpful comments

\section{Author contributions}

M.J.M., N.C. and B.K. designed the study and wrote the protocol for the whole research project entitled 'Sleep, circadian rhythmicity, mood and pain perception in patients with affective and affect regulation disorders and healthy controls'. A.-M.S. and S.F. undertook coordination and gathering of the data. K. F. B and $\mathrm{J} . \mathrm{H}$. analysed and interpreted the data, and wrote the first draft of the manuscript. All authors revised the manuscript, gave final approvement and agreed to be accountable for all aspects of the work.

\section{References}

1 Mueller MJ, Haag A. The concept of chronotypes and its clinical importance for depressive disorders. Int J Psychiat Med 2018; 53: 224-40.

2 Drennan MD, Klauber MR, Kripke DF, Goyette LM. The effects of depression and age on the Horne-Ostberg Morningness-Eveningness score. J Affect Disord 1991; 23: 93-8

3 Mueller MJ, Cabanel N, Olschinski C, Jochim D, Kundermann B. Chronotypes in patients with nonseasonal depressive disorder: distribution, stability and association with clinical variables. Chronobiol Int 2015; 32: 1343-51.

4 Gaspar-Barba E, Calati R, Cruz-Fuentes CS, Ontiveros-Uribe MP, Natale V, De Ronchi $\mathrm{D}$, et al. Depressive symptomatology is influenced by chronotypes. J Affect Disord 2009; 119: 100-6.

5 Haraden DA, Mullin BC, Hankin BL. The relationship between depression and chronotype: a longitudinal assessment during childhood and adolescence. Depress Anxiety 2017; 34: 967-76.

6 Allebrandt KV, Teder-Laving M, Kantermann T, Peters A, Campbell H, Rudan I, et al. Chronotype and sleep duration: the influence of season of assessment. Chronobiol Int 2014; 31: 731-40.

7 Roenneberg T, Wirz-Justice A, Merrow M. Life between clocks: daily temporal patterns of human chronotypes. J Biol Rhythms 2003; 18: 80-90.

8 Boland EM, Bertulis K, Leong SH, Thase ME, Gehrman PR. Preliminary support for the role of reward relevant effort and chronotype in the depression/ insomnia comorbidity. J Affect Disord 2019; 242: 220-3.

9 World Health Organization. The ICD-10 Classification of Mental and Behavioural Disorders: Diagnostic Criteria for Research (vol 2). WHO, 1993.

10 Beck AT, Steer RA, Brown GK. Beck Depression Inventory-II. Psychological Corporation, 1996: 490-8

11 Griefhan B, Künemund $C$, Bröde $\mathrm{P}$, Mehnert P. Zur Validität der deutschen Übersetzung des Morningness-Eveningness-Questionnaires von Horne und Östberg. [The Validity of a German Version of the Morningness-EveningnessQuestionnaire Developed by Horne and Östberg.] Somnologie 2001; 5: 9.

12 Horne JA, Ostberg $\mathrm{O}$. A self-assessment questionnaire to determine morningness-eveningness in human circadian rhythms. Int J Chronobiol 1976; 4: 97-110.

13 Janke W, Hüppe M, Erdmann G. Befindlichkeitsskalierung durch Kategorien und Eigenschaftswörter (BSKE). [Measurment of Emotional States by use of Kategories and Adjectives (BSKE).] Universität Würzburg, 1994.

14 Dallaspezia S, Suzuki M, Clara L, Colombo C, Benedetti F. Chronotype influences response to antidepressant chronotherapeutics in bipolar patients. Chronobiol Int 2018; 35: 1319-25.

15 Park H, Lee HK, Lee K. Chronotype and suicide: the mediating effect of depressive symptoms. Psychiatry Res 2018; 269: 316-20.

16 Rumble ME, Dickson D, McCall WV, Krystal AD, Case D, Rosenquist PB, et al. The relationship of person-specific eveningness chronotype, greater seasonality, and less rhythmicity to suicidal behavior: a literature review. J Affect Disord 2018; 227: 721-30.

17 Massing W, Angermeyer MC. The monthly and weekly distribution of suicide. Soc Sci Med 1985; 21: 433-41. 